\title{
Telescopes in the mirror of scientometrics
}

\author{
Virginia Trimble
}

Received: 24 February 2009 / Accepted: 25 February 2009 / Published online: 24 March 2009

(C) The Author(s) 2009. This article is published with open access at Springerlink.com

\begin{abstract}
Counting papers and citations is one way to estimate the significance of particular astronomical telescopes and other facilities in the long time gap between the verdict of history and the referee's report on your most recent proposal. This has been done for 2,184 observational astronomy papers published between 1960 and 1964 (with 14,237 citations in 1965-1969) and the numbers looked at in various ways. The extreme dominance of California in optical astronomy and of the UK and Australia in radio astronomy provides the background against which ESO, NOAO, NRAO, and A\&A were founded, with equality of access to facilities having increased enormously in the intervening 40 years, but inequality of results having increased slightly. A number of other factoids about astronomical publications, the community, and their environments surfaced during the counting process, and a subset reported here, including a few pertaining to the more distant past.
\end{abstract}

Keywords Scientometrics $\cdot$ Citation analysis

\section{Introduction: the astronomical world in 1960}

The time frame for this study was determined by both personal and public issues. First, I started graduate school in 1964, marking (for me!) the cut between "history" and "current events" in astronomy. Second, the first five-year compilation of citation numbers from Science Citation Index covers

\footnotetext{
V. Trimble $(\varangle)$

Department of Physics and Astronomy, University of California,

Irvine, CA 92697 USA

e-mail: vtrimble@uci.edu

V. Trimble

Las Cumbres Observatory, Goleta, CA, USA
} 
1965-1969. Third was the availability of journals-the "Russian" one in translation as Soviet AJ (though BAC had some papers in Russia (I read, but with difficulty), and the library stack contents at the Space Telescope Science Institute (STScI) and the University of California Irvine (VCI). Fourth was the rapid ramp-up of radio and space astronomy. And fifth the fading out of observatory publications as distinct from the journals we now read.

Between 1800 and about 1970, more than 200 observatories distributed their own publications-from Aarhus and Abastumai to Zo-se and Zurich (including some radio observatories) - though not all at once. At least 70 contained papers significant enough to be cited in the Kuiper compendium reviews. The L's alone included Lick (Trumpler on interstellar absorption), Lowell (Slipher's redshifts), Lund (Holmberg's binary galaxies), Liege, Lille, Leiden, Lemberg, Lembang, Lausanne, Leningrad, Lehigh, Le Hough (Peridier's private observatory, where de Vaucouleurs got his start), La Plata, Leander McCormick, Leyton, Lisbon, Lyon, London, and Louvain. An analysis of publications from the 1940s or earlier would therefore require access to one of the very few, very complete libraries of astronomy. And neither SCI nor ADS can provide lists of citations to these papers except from long after they were published. The last of these series to contain essential catalogues and such was probably that of the Dominion Astrophysical Observatory. Remarkably, quarterly issues from Skalnate Pleso in the Slovak Republic still arrive regularly.

The transition from observatory publications to broader-based journals was not entirely smooth. In 1939, Babcock was unhappy that his thesis (on the spectrum and rotation of M31) appeared as a Lick Observatory Bulletin rather than in ApJ (according to a letter he wrote when asked why he had moved so firmly away from extragalactic astronomy); and, in the same year, Popper was unhappy at his thesis being sent to ApJ when he would have preferred publication as an LOB (according to a remark he made on a conference tour bus when I mentioned the Babcock story).

The astronomical world in 1960 was, in many ways, very different from today's. Only the sun had been seen in ultraviolet, X-, and gamma rays; the AS\&E (Giaconni et al.) and NRL (Bowyer et al.) rockets that saw Sco X-1 and Crab Nebula X-rays and the Arnold and Kraushaar gamma ray background belong to our period $[3,4,7,11]$. In the radio regime, there had been very few optical identifications; $\log N-\log S$ was still disputed (in unfriendly terms!); and no QSRS's (1963), nor CMB (1965), nor pulsars (1968) had yet been found. Rapid advances in 1960-1964 occurred in mapping and polarization of the Milky Way and emission from planets including spectra; sensitivity to polarization improved as well as angular resolution; $\mathrm{OH}$ was found in the ISM; and many more extragalactic sources received optical identifications.

Women were working in all the main wavelength bands, though not in enormous numbers. Among the space pioneers were Marcia Neugebauer (direct detection of the solar wind), Phyllis Freier (cosmic rays), and W.S. Cameron (solar system from Mercury spacecraft). There were women radio astronomers in most of the countries engaged in the field: Ruby Payne-Scott (Australia), 
Patricia Leslie (UK), E.M. Berkhuijsen (Netherlands), L. Bottinelli (France), Z. Placova (Czechoslovakia), T.A. Lozinskaya (Russia), and N.H. Dieter and M.A. Kaftan-Kassim (USA). All appeared as authors, generally first authors, of papers in the current data base.

This applies also to the following women optical observers: Anne Underhill (NL \& US), A.E Ringuelet-Kaswalder, Y. Andrillat, M-C. Lortet-Zuckerman (France), S. Torres-Peimbert (Mexico), M. Jaschek (Argentina), M. Hack (Italy), Choko Fujita (Japan), Wilhelmina Iwanowska (Poland), many Americans (Margaret Burbidge, Vera Rubin, Dorrit Hoffleit, and B.T. Lynds perhaps most widely known but also Barbara Middlehurst, K.C. Gordon, Sarah Lee Lippincott, Anne Pyne Cowley, and Edith Flather), and at least 18 first authors with surnames ending in ova, skaya, etc. in the Russian, East German, Polish and Czech journals, setting a lower limit to the number of women making significant contributions there. I mention by name only A. Szczepanowska (for reasons only G.B. Shaw fans will guess).

Some other things have not changed much. There were visa issues for conferences in the USA (Dirac having been denied admission); the Kefauver committee was worried about drug prices and drug advertising; the NASA budget and the fraction devoted to space science were bouncing around (though with a peak at something like 4\% of GDP during the Apollo program that has never been equaled since); and fraudulent papers were sometimes withdrawn (e.g. Science 133, 941). The estimated cost of carrying out all the items in the Whitford report (astronomy for the 1960s) was, of course, a considerable underestimate. The initial figure, however, was only(?) \$227 million. Keep in mind, though, that typical conference registration fees were \$3-4 and hotel rooms cost \$8-15 per night, even in New York.

\section{2 (Relatively) deep time}

"Deep time" can mean thousands of years in contrast to a four-year presidential term or millions of years in contrast to the $10^{4}$ year duration of civilizations. In the present context it means items where I came across something interesting between 1608 and 1960.

The first modern astronomers, like sixteenth, seventeenth, and eighteenth century musicians, were churchmen, nobility, or royalty (remember Tycho's competitor for the parallax of SN 1572, the Landgrave of Hesse), or supported by them. They published in books and broadsheets (some shown in Van Helden's presentation) and wrote to colleagues and friends, sometimes in anagrammatical form. By the middle of the nineteenth century, there was a division between employees of government observatories and universities and wealthy amateurs (like Carrington the brewer and Draper the physician), and publication was more often in the form of articles in national academy and observatory publications. Now, of course, a large fraction of astronomers (and also of musicians) are university employees, who publish in a rather small number of weekly to monthly journals. 
The first state-supported observatories were intended to assist navigation and published almanacs and ephemerides, for instance Paris 1679 (Connoissance des Temps 1679), London 1750 (Almanac 1767), San Fernando 1757 (publications 1773), Coimbra 1772 (publications 1904), and, well behind the pack, the US Naval Observatory, founded in 1830 (Almanac 1853). Meanwhile, private observatories and ones supported by educational institutions continued to proliferate (and in the niche of British University Observatories, I recommend the recently-published thesis of conference participant Hutchins [8]). The US had, perhaps, an advantage in its multi-tiered government structure that allowed dipping into pockets of the nation, the states, and the cities.

The first scientific societies and their publications date from the same period: The Royal Society of London, founded 1660 (and publishing Philosophical Transactions from 1665) and the Paris academy and its Comptes Rendues soon after. Purely astronomical societies and publications (sometimes coupled, sometimes not) come later. There is, I think, an interesting divergence between what happened in the United Kingdom, with a single Astronomical Society of London dating from 1820 (the Royal Astronomical Society after 1831) and its Memoires (1821) and in the divided areas called Germany and Italy, where there were very many local observatories, and the first journal (and now the oldest one in continuous publication, Astronomische Nachrichten, founded in 1821) was an extension of the letters-to-distant friends system, invented by Heinrich Christian Schumacher. He asked European astronomers to send him their letters in any European language; he then edited and assembled these and sent them back to everybody).

The RAS illustrates a very general principle that it takes about 100 people to establish a viable society with some sort of paper trail and regular meetings. Other organizations where you are like to find astronomer and for which this was true include the American Association for the Advancement of Science (1850, initially naturalists, geologists, and astronomers), the Italian society (1872, focused on spectroscopy and so arguably the first astrophysical society), Royal Astronomical Society of Canada (1868), Belgium (1880), France (1887), Astronomical Society of the Pacific (1895), American Physical Society, (1898), American Astronomical Society (1899), and the International Astronomical Union (1919).

National astronomical societies and some associated journals proliferated just before and just after World War I (Japan 1908, South Africa 1922, China 1922, New Zealand 1920, Poland 1925, Netherlands, 1921, USSR, 1924, and the Scandinavian journal Arkiv 1916). All of these survive in some form. Realignments after 1990 have added separate societies in the Czech and Slovak republics and parts of the former Yugoslavia and USSR, some with perhaps subcritical membership. Additional details of some of these "community" issues appear in [16].

Also stretched out over the entire four centuries is the gradual incorporation of women into astronomy. This occurred, I think, in three slightly overlapping stages. At first, the only choice was working with a father, husband, or brother. Caroline Herschel is the best known of these, but Sophia Brahe, Elizabeth 
Hevelius, and Margaret Huggins are other examples. Next come formal, institutional helpers, the "computers" (also found in laboratories looking at cloud chamber tracks and in other projects with large quantities of data to extract or classify). Henrietta Swan Leavitt, Annie Jump Cannon, Williamina Paton Stevens Fleming, Antonia Caetana de Paiva Pereira Maury, and Donna Ebert (who worked with S. Chandrasekhar) on the theoretical side are astronomical examples of women whose work was important enough and independent enough that we remember them by name. The last of the traditional Mt. Wilson computers were still on duty when I started graduate school at Caltech in 1964. Finally comes the stage of full participation in research science, where the (female) astronomer asks a question, figures out how to answer it, and presents the work to her colleagues. Cecilia H. Payne (later Gaposchkin) was arguably the first of these with her 1925 thesis showing that virtually all stars have about the same chemical composition (and that $\mathrm{H}$ and He dominate). Bridgers across the phases include Maria Mitchell (who began working with her father, was a computer at USNO, and ended as professor at Vassar) and Ethel F. Bellamy of Oxford, who began working with an uncle, was a computer under Turner, and acquired solo responsibilities during World War II when most of the men were called to war service.

We end this section with Table 1 , which shows total citation numbers in 1965-1969, first, to the works of four famous telescope pioneers; second, to five papers between 1912 and 1939 that I think were enormously important and did not appear in the journals now of high prestige (notice that John Herschel trumps William, and that only Trumpler's interstellar absorption left much of a footprint during this period); and third, to nine papers published in ApJ or AJ between 1953 and 1965 and singled out as highlights in the centenary

Table 1 Some giants' shoulders, citations 1965-1969

\begin{tabular}{lr}
\hline Galileo (all) & 50 \\
Huygens (all) & 19 \\
Wm Herschel & 17 \\
John Herschel & 22 \\
Slipher galaxy spectra (1912-1920) Lowell & 4 \\
Shapley Mt. W. Contr. 1918 & 7 \\
Payne 1925 Harvard Monograph & 1 \\
Hubble Proc. NAS 1929 & 3 \\
Trumpler 1930 Lick $A_{\mathrm{v}}=1^{\mathrm{m}} / \mathrm{kpc}$ & 30 \\
Zwicky 1933 Helv. Ph. Acta DM & 5 \\
Babcock M31 rotn Lick 1939 & 5 \\
Johnson \& Morgan color system '53 & 184 \\
Babcock solar mag. Cycle '53 & 29 \\
HMS H = 180 1956 & 160 \\
Wilson/Bappu effect 1957 & 46 \\
Abell 1958 clusters of Gs & 34 \\
Eggen L-B S, MW history 1962 & 49 \\
Neugebauer +'65, IR YSO cat. & 55 \\
Penzias \& Wilson 1965 & 345 \\
Gunn-Peterson '65 & 59 \\
Hewish, et al., Bell pulsars 1968 & 99 \\
\hline
\end{tabular}


volume edited by Abt [1]. Hewish et al. on pulsars is tucked at the end just to show that it is possible to accumulate many citations quickly. While two of the highly cited papers are cosmological (Penzias \& Wilson finding the CMB and Humason, Mayall, \& Sandage correcting the Hubble constant to a value of $180 \mathrm{~km} / \mathrm{s} / \mathrm{Mpc}$ ) the third is Johnson \& Morgan on color systems for stars.

\section{The 1960-1964 papers and 1965-1969 citations}

This section and the next are mostly about decisions: which journals to consult, which papers to count, which facilities to keep track of, and how to apportion papers and citations among facilities when more than one contributes to a paper. Which journals was entirely a matter of availability: ApJ (and Supplements), AJ, PASP; those that in 1969 folded into A\&A (AN, Zs f Ap, Ann d'Ap, BAN, and BAC, with Bull. Astron., J. Obs., and Arkiv not available), MNRAS, Observatory, Astron. Zh (= Sov. AJ), Acta Astron., Nature, Phys. Rev. Letters, Science, PASJ, Icarus, and Australian Journal of Physics (the radio physics part). In the process of looking up citation numbers for these, I learned that some authors of papers in these journals had also published observational papers in physics and geophysics journals, in academy proceedings, and in observatory publications, but for none of them these most of the papers or (with a couple of very senior exceptions) their most cited.

As for which papers to count, I left out meeting abstracts (which appeared in AJ, PASP, Observatory, and Ann. d'Ap. regularly) but included meetings proceedings papers. In the days before Astro-ph and all, a few of these abstracts were cited a dozen or more times in 1965-1969, but, again, not many. Otherwise, every paper, even one-page ones, was included if it reported or analyzed observational data at any wavelength (including cosmic rays) and the telescope(s) used could be identified.

Table 2 shows the distribution by journals of numbers of papers, citations, and citations per paper, largest citation numbers, and numbers of papers with zero citations in each journal. This last addresses the claim "most papers aren't read by anybody." In fact, $22 \%$ went uncited (vs. $6 \%$ in recent data), but these are concentrated in five or six journals. Citation numbers were most difficult to determine for Sov. AJ. SCI recorded citations for both the Russian and translation versions, but the volume numbers, page numbers, and even years are not the same. I think, though, I caught them all. Self-citations are included for all papers, and one author (no, I won't tell you who) had only self-citations in the quinquennium.

What was an appropriate set of subtopics to look at? The choice was to use the same ones as in [17] and earlier papers in that series to facilitate comparison, though the result was one white dwarf paper (by Volker Weidemann), two on exoplanets (Peter van de Kamp reporting false alarms around Barnard's star), none on GRBs, and something of a pile-up under "stars." Topic assignments sometimes required a bit of 20-20 hindsight to decide, for instance, whether a set of radio angular diameters pertains to SNRs or AGNs. 
Table 2 Distribution by journals of number of papers

\begin{tabular}{lcrrcc}
\hline Journal & Citations & Papers & \multicolumn{1}{c}{$C / P$} & No. 0's (\%) & Largest C's \\
\hline ApJS & 377 & 21 & 17.95 & $2(10)$ & 52,43 \\
Aust J. Ph. & 241 & 15 & 16.07 & $1(7)$ & 88,45 \\
PRL & 958 & 66 & 14.52 & $12(18)$ & 85,57 \\
Nature & 1,603 & 192 & 8.35 & $37(20)$ & 65,64 \\
Science & 463 & 72 & 6.42 & $18(25)$ & 72,60 \\
ApJ & 4,964 & 500 & 9.90 & $43(9)$ & 139,109 \\
AJ & 1,341 & 222 & 6.04 & $46(21)$ & 50,44 \\
PASP & 545 & 234 & 2.33 & $82(35)$ & 39,30 \\
Icarus & 203 & 19 & 10.68 & $1(5)$ & 38,30 \\
MNRAS & 1,426 & 147 & 9.70 & $14(10)$ & 137,50 \\
Observ. & 176 & 94 & 1.87 & $32(34)$ & 12,10 \\
BAN & 338 & 47 & 7.19 & $8(17)$ & 51,25 \\
Ann d'Ap & 378 & 75 & 5.04 & $13(17)$ & 54,29 \\
Zs f Ap & 197 & 67 & 2.94 & $18(27)$ & 30,23 \\
PASJ & 270 & 67 & 4.03 & $15(22)$ & 14,14 \\
A Zh/Sov AJ & 622 & 173 & 3.60 & $50(29)$ & 32,31 \\
Acta Astr. & 47 & 45 & 1.04 & $29(64)$ & 9,5 \\
BAC & 45 & 50 & 0.90 & $20(40)$ & 9,8 \\
AN & 43 & 58 & 0.74 & $33(57)$ & 6,6 \\
Total & 14,237 & 2,164 & 6.58 & $474(22)$ & \\
\hline
\end{tabular}

The recent data excluded solar astronomy, which uses a nearly disjoint set of telescopes etc., but for 1960-1964 it turned out that radio and space astronomy had a very large component focused on the sun, heliosphere, magnetosphere, and sun-earth connections, and that these papers were the break-outs into Nature, Science, and Phys. Rev. Letters. They were thus included as separate topics: sun-earth radio and sun-earth space. "Service," incidentally, means catalogues, coordinate systems, and such.

Table 3 shows numbers of papers and citations by subfield, but notice that only the 1,248 papers (a bit more than half the total) with at least four citations in 1965-1969 are included. Those papers averaged 13.8 citations per paper

Table 3 Papers with $\geq 4$ citations in 5 years

\begin{tabular}{lrrr}
\hline Category & \multicolumn{1}{c}{$C$} & \multicolumn{1}{c}{$P$} & $C / P$ \\
\hline Radio (solar-earth) & 294 & 32 & 9.2 \\
Radio other & 4,083 & 305 & 13.4 \\
Space (solar-earth) & 669 & 44 & 15.2 \\
Space other & 1,172 & 53 & 22.1 \\
Optical all & 10,998 & 814 & 13.5 \\
Optical service & 374 & 21 & 17.8 \\
Cosmology & 513 & 24 & 21.4 \\
Active galaxies & 1,522 & 55 & 27.7 \\
Other extragalactic & 896 & 89 & 10.1 \\
MW, ISM, YSO & 1,636 & 126 & 13.0 \\
NS/BH/SNe/SNR/CRs & 898 & 48 & 18.7 \\
Solar system & 2,162 & 169 & 12.8 \\
Exoplanets (vdK) & 16 & 2 & 8.0 \\
WDs (Weidemann) & 9 & 1 & 9.0 \\
Stars, star clusters, CBs, CVs, PNe & 2,896 & 267 & 10.8 \\
\hline
\end{tabular}


over the 5 years. In comparison with the average, cosmology, active galaxies (meaning both QSRS and radio galaxies), and neutron stars, supernovae, etc. and cosmic rays (for which the electron component was finally found in this period) count as hot topics; solar system and Milky Way are about average; and stars are already an endangered species. Coming down to 2001-2006, the solar system and stars have become less popular (in paper numbers and $\mathrm{C} / \mathrm{P}$ ), the Milky Way has roughly held its own, and extragalactic topics have increased both their fraction of the total astronomical literature and their relative citedness.

\section{Facilities}

How many telescopes (etc) contributed to the literature, and which ones did I keep track of individually? In 1960 there were, say Kuiper and Middlehurst [12], 100 sites with at least one optical telescope of $20^{\prime \prime}$ or larger (a total of 155 such telescopes), and I kept track of each individually, though many have been summed in the following table. The KPNO 36", Japanese $1.88 \mathrm{~m}$, and others gathered their first photons during the period and were added to the ones tracked. There were also something like 200 smaller telescopes whose data appeared in at least one paper, and these were associated either with observatories having larger telescopes or grouped by geographical regions. Curiously, the total number of optical telescopes contributing to data published in 2001-2003 is not enormously larger.

The radio sites numbered about 75, only a subset appearing in [12] with perhaps 100 widgets. Cambridge most notably built and dismantled rapidly, though younger colleagues assure me that saying only ASA 800 film could be used to photograph them (hence the relatively poor images in both Sullivan's presentation and mine) will not be understood by their generation. Most of the radio telescopes in 1960 were in the UK and Australia; then the US roars in (as we seem to have been doing since at least 1917). There were European facilities at Nançay and Dwingeloo, Ondrejov and Bonn. And very many places engaged in only solar radio astronomy, e.g. Pisa, Cracow, Torun, Belgrade, Meudon, Berlin, Arcetri, Kiruna, Slough, Aldershot, Oslo, Nagoya, and Sagamore Hill. Again the total numbers 40 years later were only about $20 \%$ larger.

In space, very few things were used more than once. There were lots of balloons and aircraft; very many rockets (mostly Aerobees but also Skylarks, Journeymen, Atlas, and Veronique); and satellites with other primary purposes that turned out to be doing astronomy, including multiple Rangers, Luniks, Pioneers, Sputniks, Discovers, Vanguards, IMP, Injun, Mercury, Mariner, Alouette, Telstar, Echo, and Transit. This list has all the devices that contributed to the 1960-1964 papers and also some from Seaborn [13], which lists all of the space project launches (including failures) from 1957 to 1967 and is undoubtedly the most valuable free publication I ever picked up off an 
exhibitor's table! By the end of 1964, 51 Kosmos satellites and four Vela's had been launched, but we did not yet know that they were doing astronomy!

Over the next 40 years, the most conspicuous change was the rise of dedicated astronomical satellites. Solar (OSO) and ultraviolet (OAO) ones came first, but Uhuru in 1971 is the one you are perhaps most likely to remember or have heard of, right on down to Fermi (the republic formerly known as GLAST). For what it is worth, I thought the space X- and gamma-ray talks said too little about MIR/KVANT and other aspects of the Soviet program, but then so did I, for there were no "space" papers in Sov. AJ. MIR/KVANT of course belongs to a later period, as do even OSO-2 (Feb. 1965 launch and observation of solar UV, X, and gamma-rays) and OAO-2 (December 1968 launch for stellar UV observations).

\section{Papers and citation numbers by telescope (etc...)}

At this point we need (a) to do a bit more work, looking up citation numbers for 1965-1969 for all the 1960-1964 papers recovered from the journals listed in Section 3 and (b) to make another decision-how to apportion credit among the telescopes when more than one contributed to a given paper. Folks who care about a single telescope generally give it full credit for every paper that uses its data, but we adopt here the same algorithm described in [17]. Space, radio, and optical bands are considered separately, but within each band, all telescopes contributing receive equal partial credit for both the paper and the citations: Thus a paper with data from the Palomar $48^{\prime \prime}$ Schmidt, the $200^{\prime \prime}$, and the Mt. Wilson $100^{\prime \prime}$ with 24 citations counts as one third of a paper and eight citations for each. If $C / N$ is not an integer ( 25 citations and three telescopes, for instance), the extra one(s) go to the first telescope(s) mentioned by the authors, thus in the hypothetical example the Schmidt would get nine and the other two telescopes eight each.

Table 4 presents the optical numbers and Table 5 the radio ones. There is binning of multiple small telescopes on a single site and even by geographical area to keep the tables manageable. The "widget" class includes an early Ray Davis limit on solar neutrinos, Robert Dicke's solar oblateness measurements, and the Hanbury Brown interferometer at Narrabri. I separated high-altitude sites (Haleakala, White Mountain, Pic du Midi, Chalcultaya) to see whether the extra effort required to operate there paid off. The answer would seem to be yes, but there are only 18.5 papers involved.

There are no major surprises to be found in these tables. The Mt. Wilson $100^{\prime \prime}$ yielded the most optical papers, but the Palomar 200" the most-cited ones. Similarly at radio wavelengths, Jodrell and Australia produced more papers but Cambridge the most-cited ones. Overall, radio papers were cited almost twice as often as optical ones. This was not true for 2001-2003, which led to some awkwardness between authors and referees presumably from the radio community. 
Table 4 Optical papers and citations, pro-rated

\begin{tabular}{lrrrrl}
\hline Facility & Citations & Papers & $C / P$ & $N_{\max }$ & First light \\
\hline Palomar & & & & & \\
$200^{\prime \prime}$ & 1,252 & 88.7 & 14.1 & 139,100 & 1948 \\
$48^{\prime \prime}$ & 459 & 79.9 & 5.7 & 69,35 & 1948 \\
$\quad$ Other & 170 & 17.2 & 9.9 & 34,22 & \\
Mt. Wilson & & & & & \\
$\quad 100^{\prime \prime}$ & 1,127 & 164.9 & 10.8 & 89,74 & 1917 \\
$60^{\prime \prime}$ & 330 & 37.2 & 8.9 & 103,25 & 1908 \\
$\quad$ Other & 10 & 9.8 & 1.0 & 6,2 & \\
McDonald & & & & & \\
$82^{\prime \prime}$ & 415 & 79.6 & 5.2 & 29,27 & 1939 \\
Other & 178 & 20.3 & 8.8 & 25,25 & \\
Lick & & & & & \\
$\quad 120^{\prime \prime}$ & 261 & 49.6 & 5.3 & 34,24 & 1959 \\
Crossley & 225 & 42.4 & 5.3 & 44,20 & 1898 \\
Other & 90 & 28.0 & 3.2 & 15,7 & \\
Arizona & 301 & 56.1 & 5.4 & 24,21 & \\
Other US & 593 & 162.2 & 3.7 & 61,40 & \\
Canada & 105 & 23.2 & 4.5 & 10,10 & \\
South Africa & & & & & \\
$\quad 74^{\prime \prime}$ Radcliffe & 224 & 49.9 & 4.5 & 29,16 & 1948 \\
Other & 248 & 64.1 & 3.9 & 21,20 & \\
Stromlo (etc.) & & & & & \\
$\quad 74^{\prime \prime}$ & 144 & 36.4 & 4.0 & 16,11 & 1955 \\
Other Aust. & 169 & 44.8 & 3.8 & 18,11 & \\
USSR & 346 & 108.1 & 3.2 & 47,34 & \\
E. Europe & 109 & 109.7 & 1.0 & 10,9 & \\
W. Europe & 422 & 118.9 & 3.6 & 35,30 & \\
Asia, So. Am. etc. & 94 & 40.6 & 2.3 & 11,10 & \\
High altitude & 293 & 18.5 & 15.8 & 43,29 & \\
Widgets & 119 & 4.0 & 29.7 & 57,51 & \\
TOTAL & 7,684 & 1,454 & 5.8 & & \\
\hline
\end{tabular}

Comparison of these numbers with 21st century ones has something to tell us about changes in equality of opportunity and achievement. In each time frame, the top seven or eight optical telescopes yield about $40 \%$ of the papers and $50 \%$ of the citations, but their geographical distribution is very different. All the early high achievers were in the USA, and California alone was responsible for half the citations and a third of the papers. Now the high-productivity telescopes are scattered among Chile, Hawaii, the Canary Islands, Australia, and outer space, and nearly all of the world's astronomers can apply for time on many of them, while Lick, Mt. Wilson, and Palomar had very restricted user communities. This casts some light on what Allan Sandage meant, in an oral history discussion of steady state cosmology [10]. When he said that he didn't think steady state was ever taken very seriously in California, he was referring not to theorists but to observers with access to the large telescopes.

The top $1 \%$ of citation-gatherers are listed in Table 6 . They include space, radio, and optical studies, most from well-known facilities, and have garnered $12 \%$ of all the citations. The top $1 \%$ of the papers for $2001-2003$ are again spread over all wavelengths, come mostly from well-known (and well 
Table 5 Radio: All papers and citations pro-rated

\begin{tabular}{|c|c|c|c|}
\hline Facility/location & Citations & Papers & $\mathrm{C} / \mathrm{P}$ \\
\hline Jodrell & 446 & 41.97 & 10.6 \\
\hline Cambridge & 592 & 35.34 & 16.8 \\
\hline Australia & 623 & 41.94 & 14.9 \\
\hline Other European ${ }^{1}$ & 307 & 44.37 & 6.9 \\
\hline NRAO & 272 & 33.39 & 8.2 \\
\hline OVRO & 858 & 35.81 & 24.0 \\
\hline NRL & 120 & 20.0 & 6.0 \\
\hline Harvard & 134 & 17.0 & 7.9 \\
\hline Other US ${ }^{2}$ & 592 & 50.4 & 11.7 \\
\hline Canada, NZ, UK & 80 & 14.0 & 5.7 \\
\hline USSR & 204 & 48.9 & 4.5 \\
\hline Total & 4,228 & 383.1 & 11.0 \\
\hline
\end{tabular}

(1) Dwingeloo, Nançay, Ondrejev, Bonn, (2) Goldstone, U. Mass, U. Mich, Ohio State, MIT, Florida State, Yale, Boulder, Clark Lake, others, (3) Ottawa, DRAO, Algonquin, Malvern, Christchurch. In addition, many places were doing solar radio: Pisa, Cracow, Torun, Belgrade, Meudon, Berlin, Arcetri, Kiruna, Slough, Aldershot, Oslo, Nagoya, Sagamore Hill. US papers are concentrated in later years. About 100 devices on 80 sites

supported) facilities; and are responsible for $15 \%$ of the citations. Thus while equality of access has improved over the 40 intervening years, achievement has become a bit more unequal.

Table 7 lists the most-cited paper from each of 23 lower-profile astronomical telescopes, perhaps illustrating the principle mentioned by other speakers that the right people with the right ideas are the most important astronomical

Table 6 The most cited papers (1\%)

\begin{tabular}{lllll}
\hline$N$ & Journal & First author & Facility & Subject \\
\hline 139 & ApJ & Greenstein & $200^{\prime \prime}$ & Quasars \\
137 & MNRAS & Conway & Cambridge, Jodrell, OVRO & Quasars \\
109 & ApJ & Matthews & OVRO, 200, 60, POSS & Quasars \\
103 & ApJ & Oke & Mt. W. 60 & Calibrations \\
100 & ApJ & Sandage & $200^{\prime \prime}$ & $\mathrm{H}_{\text {O }}$ \\
96 & ApJ & Kellermann & OVRO & Radio sources \\
89 & ApJ & Kaplan & Mt. W. 100" & Mars atm. \\
88 & Aust. JP & Bolton & Parkes, POSS, Stromlo 74" & Catalogue \\
85 & PRL & Giacconi & Aerobee & Sco X-1 \\
83 & ApJ & Matthews & POSS $<200^{\prime \prime}, 120^{\prime \prime}$, Stromlo 74" & AGN \\
72 & Science & Bowyer & Aerobee & Crab \\
65 & Nature & Schmidt & $200^{\prime \prime}$ & 3 C273 \\
64 & Nature & Greenstein & $200^{\prime \prime}$ & 3 C48 \\
63 & Nature & Hewish & Cambridge & Scintillation \\
61 & ApJ & O'Dell & Pine bluff & PNe \\
60 & Science & Neugebauer, M. & Mariner II & Solar wind \\
59 & ApJ & Maltby & OVRA, POSS & Radio sources \\
58 & ApJ & Heeschen & NRAO 300' & Radio galaxies \\
58 & Nature & Bowyer & Aerobee & Crab, Sco X-1 \\
57 & PRL & Davis & $C_{2}$ Cl tank & Nu limit \\
54 & Ann d'Ap & Lequeux & Nancay & Radio sources \\
54 & PRL & Ness & IMP-1 & IPM \\
\hline
\end{tabular}

1754 or $12 \%$ of all citations to top $1 \%$ papers vs $13-15 \%$ for $2001-2003$ 
Table 7 Significantly cited papers from less obvious telescopes

\begin{tabular}{lllll}
\hline Number $(N)$ & Journal & First author & Facility & Subject \\
\hline 61 & ApJ & O'Dell & Pine bluff & PNe \\
54 & Ann d'Ap & Lequeux & Nancay & Radio sources \\
44 & AJ & Kron & Crossley 36" & Star clusters \\
43 & Nature & Metzger & Ranger 5, 3 & Gamma bkgd. \\
38 & AJ & Gehrels & Goethe Link 36" & Solar system \\
38 & AJ & Eggen & Cape 24", 18" & Stars \\
31 & Nature & Smith, HJ & Harvard patrol & 3C 273 \\
30 & Sov AJ & Moroz & Sternberg 1.25m & Solar system \\
29 & MNRAS & Feast & Radcliffe 74" & Galaxies \\
29 & MNRAS & Ingham & Chacultaya & Solar system \\
29 & AJ & Eggen & Cape 24" 18" & Milky way \\
29 & ApJ & Murray, BC & White Mt. 19" & Solar system \\
28 & MNRAS & Griffin, RF & Cambridge 36" & Stars \\
27 & Ann d'Ap & Courtès & OHP 1.93 & Supernovae \\
25 & ApJ & Brandt & McD 10" & Galaxies \\
25 & BAN & Van Woerden & Dwingeloo & Milky way \\
25 & Ann d'Ap & Weinberg & Haleakala & Solar system \\
24 & ApJ & Slettebak & Perkins 69" & PNe \\
23 & ApJ & Collins & Washburn & PNe \\
24 & Sov AJ & Basharinov & 8 mm something & Solar system \\
24 & AJ & Zabriskie & Slope Obs. & Solar system \\
23 & Zs f Ap & Elsässer & Boyden 30 cm + Jungfraujoch & Milky way \\
22 & MNRAS & Westerlund & Uppsala So. Sch. & Stars \\
\hline
\end{tabular}

facilities. For what it is worth, three of Tables 6 and 7 first authors were at the meeting '400 years of Astronomical Telescopes': Giacconi, Lequeux, and O'Dell. But the Gehrels of the Table is the father of participant Gehrels.

The binning of years and facilities has concealed many details. One of my favorites might be called "Hit the ground running?" Among the radio telescopes (etc.) represented, many first came on line during the quinquennium and so contributed nothing the first year or two and a great deal toward the end. Table 8 shows seven examples. The numbers are citations to the papers written during the individual target years. Parkes and Owens Valley are the most spectacular, but each of the seven seems to have got up and running very quickly. Optical telescopes today seem to take longer to shake down according to Table 9. The numbers are papers rather than citations and would have been zero for Gemini, HET, Magellan, and Subaru a year or two before, but not

Table 8 Hit the ground running? Total number of citations in 1965-1969 to papers making use of data from the following facilities, published in individual years

\begin{tabular}{llrrrr}
\hline Facility & 1960 & 1961 & 1962 & 1963 & 1964 \\
\hline Parkes & 0 & 10 & 36 & 72 & 237 \\
Jodrell 250' & 9 & 39 & 120 & 179 & 120 \\
NRAO 300' & 0 & 0 & 0 & 22 & 105 \\
OVRO Interf. & 0 & 12 & 167 & 165 & 468 \\
Goldstone & 0 & 0 & 10 & 7 & 106 \\
Arecibo & 0 & 0 & 0 & 0 & 6 \\
Ohio State Monst. & 0 & 0 & 0 & 1 & 30 \\
\hline
\end{tabular}


Table 9 Optical ramp-up comparison: Numbers of papers (pro-rated) attributable to data from new facilities by year

\begin{tabular}{lrrr}
\hline Facility & \multicolumn{1}{c}{2001} & 2002 & 2003 \\
\hline Gemini & 2.2 & 15.2 & 19.0 \\
HET & 1.5 & 4.8 & 8.2 \\
Magellan & 4.0 & 5.4 & 18.2 \\
Subaru & 18.7 & 40.5 & 19.9 \\
VLT & 69.8 & 106.7 & 169.1 \\
Keck & 104.5 & 129.7 & 131.4 \\
\hline
\end{tabular}

for the VLT and Keck, which were in the process of increasing the effective numbers of mirrors available to the community.

\section{Other changes and other topics one might explore}

Any rational being who looks at science publishing over the 20th century will be most struck by "MORE!!!" More papers, more authors, more facilities, though fewer astronomical journals with the demise of observatory publications and the A\&A merger. I haven't counted authors but there were 2,164 observational papers in 1960-1964 (433 per year, including solar-terrestrial radio and space ones) and 11,831 papers in 2001-2003 (3944 per year, excluding all solar work). More people and papers are not, of course, unique to astronomy. The founders of Physical Review Letters noted that in 1925, Zs. f. Physik, arguably the most prestigious of the time, had 285 papers by 367 authors, while the 1963 PRL had 1600 papers (and longer ones) by 2500 authors. The weekly PRLs of 2008 are on track to add up to more than 4,000 papers, and the Phys. Rev. total will be about 18,000 [14], with author numbers dominated by collaborations of 100 and more people, generally listed alphabetically.

Within astronomy, the fraction of single-author papers has declined from more than $50 \%$ to about $10 \%$ and the fraction of multi-national papers increased from 5\% to $40 \%$. Abt [2] shows that these trends are common to many sciences, though astronomy is uniquely multinational, perhaps because the sky is there $24 / 7$ both north and south; and the unique structure of the IAU may have played a role (the other international unions have only nations and societies as members, vs. our more than 9,000 individual colleagues). Multiwavelength papers have gone from rare (1.2\% in 1960-1964) to common $(26 \%$ in 2001-2003).

The average lengths of papers have climbed more or less monotonically by factors of two to five from 1900 into the 1980s in physics, math, chemistry and astronomy, and in the UK and Japan as well as in the US [15]. That trend continued in astronomy for another decade at least. Leveling off has been claimed, but I suspect proper allowance has not been made for the increasing range of tabular, graphical, and mathematical material that now appears online only. The average ApJ Letter is now longer than the average paper of the inter-war years; and no one can now claim to read "all" of anything.

Astronomy today is a sort of economic indicator, in the sense that the ratio of GDP of a country to its number of IAU members is bracketed within 
relatively narrow limits, which can be checked by anybody with an almanac (e.g. [5]) and an IAU membership directory (e.g. [6]). Let us take as our unit billions of \$US in GDP per IAU member. Then the average (with very small dispersion) for 23 developed country members is 4.81 ; for 12 developing countries 6.86; for 15 members from Eastern Europe and the former Soviet Union 1.26; and for eight Moslem countries 16.1. For what it is worth, Israel at 1.86 resembles the places from which many of its immigrants came and South Korea and Taiwan at 10.5 and 10.2 resemble the Moslem group of rather prosperous countries with little tradition of astronomy. Notice that we have hereby discovered the obvious by a very difficult method!

Anyone with a set of old almanacs and past IAU membership directories (these go back to 1923 or so and appear in Transactions B) could find out whether the cost per astronomer has inflated faster or slower than the general consumer price index. If anyone decides to do this, I would very much like to know the answer. So, perhaps, would your Office of Management and Budget.

There have been, of course also enormous changes in the identities of the telescopes contributing to published data. Nothing that penetrated into space appears in both 1960-1964 and 2001-2003. At radio wavelengths, the Jodrell $250^{\prime}$, the Parkes $230^{\prime}$, the OVRO interferometer, Dwingeloo, Nançay, and Lebedev appear in both data sets (but all after varying degrees of modification); and a number of older sites, like Goldstone and MIT's Millstone Hill Radar installation, have almost complete new facilities. Both were originally designed to keep in touch with man-made stuff in space.

Optical longevity is also considerable. Although Mt. Wilson is gone from the journals, the Palomar $48^{\prime \prime}$ Schmidt remains remarkably productive. The large mirrors at Palomar and Lick and the McDonald 82" are still with us. Of the 72-74" telescopes, the Radcliffe in South Africa remains its most productive research instrument; others at Mt. Stromlo, DDO, DAO, and in Japan carry on at some level. And a 2008 press release says that the Perkins 69" (moved from Ohio to Arizona in 1961) is getting a new spectrophotometer and such. Particular telescopes at OHP, Pic du Midi, KPNO, Bosque Alegre, Asiago, Lowell, and in the Crimea are still yielding data for publication. And, of course, the telescope everyone loved to hate, the Great Melbourne, provided a few papers in 1960-1964 in its 1954 MSSO incarnation, and MACHO papers 40 years later, until fire swept the area in our own decade. There were also some telescopes larger than $20^{\prime \prime}$ that did not contribute any papers during either period. Some were in places with awkwardly bright skies (Lisbon, Rome) and others in places difficult for other reasons. Indeed the only instrument I failed to trace at all is a $38.5^{\prime \prime}$ multi-focus instrument that was built in what was then Elizabethville, Congo in 1960 [12].

Acknowledgements My hearty thanks go to many folks: George Herbig, Paul Hodge, Patricia Whitelock, Michael Feast, Patricia Henning, and John Mathews for help in chasing down some relatively obscure telescopes; Jet Katgert for showing all the images (some borrowed from references [9] and [12]) while I was speaking at the conference and for thoughtful, friendly, but firm discussion of the text; librarians Jeanine and Jill at UCI and STScI for help beyond the call of 
duty in accessing paper copies of all the 1960-1964 journals; and the SOC and LOC not only for the invitation but for agreeing to accept this contribution typed on paper!

Open Access This article is distributed under the terms of the Creative Commons Attribution Noncommercial License which permits any noncommercial use, distribution, and reproduction in any medium, provided the original author(s) and source are credited.

\section{References}

1. Abt, H.A. (ed.): AAS centennial volume. Astrophys. J. 525(1C), Part 3 (1999)

2. Abt, H.A.: Scientometrics 72, 156 (2007). doi:10.1007/s11192-007-1686-z

3. Arnold, J.R., et al.: J. Geophys. Res. 67, 4878 (1962). doi:10.1029/JZ067i012p04878

4. Bowyer, S., et al.: Nature 201, 1307, Science 146, 912 (1964)

5. Economist: Pocket World in Figures. Profile Books (2007)

6. Engvo1d, O. (ed.): Trans. IAU 25B. Astronomical Society of the Pacific (2007)

7. Giacconi, R., et al.: Phys. Rev. Lett. 9, 439 (1962). doi:10.1103/PhysRevLett.9.439

8. Hutchins, R.: British University Observatories 1772-1939. Ashgate Publications (2008)

9. King, H.C.: The History of the Telescope. Charles Griffith \& Co. (1955)

10. Kragh, H.: Cosmology and Controversy. Princeton University Press (1996)

11. Kraushaar, W.L., Clark, G.W.: Phys. Rev. Lett. 8, 106 (1962). doi:10.1103/PhysRevLett.8.106

12. Kuiper, G.P., Middlehurst, B.M. (eds.): Telescopes. Univ. Chicago Press (1960)

13. Seaborn, H.T. (ed.): TRW Space Log 7(4), (1967-68)

14. Sprouse, G.D.: Phys. Rev. Lett. 101, 120001-1 (2008). doi:10.1103/PhysRevLett.101.120001

15. Trimble, V.: Publ. Astron. Soc. Pac. 96, 1007 (1984). doi:10.1086/131467

16. Trimble, V.: BeamLine 32(1), 42 (2002)

17. Trimble, V., Ceja, J.A.: Astron. Nachr. 329, 632 (2008). doi:10.1002/asna.200810999 